\title{
SIMPLE SEQUENCE REPEATS MINING USING COMPUTATIONAL APPROACH IN CHLOROPLAST GENOME OF MARCHANTIA POLYMORPHA
}

\section{ПОИСК ПРОСТЫХ НУКЛЕОТИДНЫХ ПОВТОРОВ В ХЛОРОПЛАСТНОМ ГЕНОМЕ MARCHANTIA POLYMORPHA С ПОМОЩЬЮ КОМПЬЮТЕРНОЙ ПРОГРАММЫ}

\author{
ASHEESH SHANKER ${ }^{1}$ \\ АШЕЕШ ШАНКЕР ${ }^{1}$
}

\begin{abstract}
Simple sequence repeats (SSRs) or microsatellites are found in DNA sequences and consist of short repeat motifs of 1-6 bp. These repeats play important role in the development of molecular markers, phylogenetics, population genetics and evolutionary biology. The present analysis was conducted to detect chloroplastic SSRs (cpSSRs) in Marchantia polymorpha. The chloroplast genome sequence of $M$. polymorpha was downloaded from the National Center for Biotechnology Information (NCBI) and mined with the help of a Perl script named MISA. A total of $66 \mathrm{cpSSRs}$ were detected in $121.024 \mathrm{~kb}$ sequence mined showing density of $1 \mathrm{SSR} / 1.83 \mathrm{~kb}$. Depending on the repeat units, the length of SSRs ranged from 12 to $17 \mathrm{bp}$ for mono, 12 to $64 \mathrm{bp}$ for di, 12 to $21 \mathrm{bp}$ for tri, 12 to $24 \mathrm{bp}$ for tetra, $15 \mathrm{bp}$ for penta and $18 \mathrm{bp}$ for hexa nucleotide repeats. Mononucleotide repeats were the most frequent repeat type $(42.42 \%)$ followed by dinucleotide $(25.76 \%)$ and tetranucleotide $(21.21 \%)$ repeats. PCR primers were successfully designed for 45 (68.18\%) cpSSRs of M. polymorpha.
\end{abstract}

Резюме

Простые нуклеотидные повторы (SSRs), или микросателлиты, встречающиеся в последовательностях ДНК, состоят из коротких повторов из 1-6 нуклеотидов. Они важны для разработки молекулярных маркеров для филогенетических, популяционно-генетических и эволюционных исследований. Представленный анализ проведен с целью определения хлоропластных SSR Marchantia polymorpha. Использован хлоропластный геном M. polymorpha из ГенБанка и в нем проведен поиск с помощью оригинального скрипта MISA на языке Perl. Выявлено 66 SSR маркеров в последовательности длиной $121024 \mathrm{bp}$; таким образом, имеется $1 \mathrm{SSR}$ повтор на $1.83 \mathrm{kbp}$. Длина SSR повторов варьирует от 12 до 17 bp для моно-, 12 до 64 bp для ди-, 12 до 21 bp для три-, 12 до 24 bp для тетра-, 15 bp для пента- и 18 bp для гекса-нуклеотидных повторов. Мононуклеотидные повторы наиболее часты (42.42\%), реже встречаются ди- $(25.76 \%)$ и тетра- нуклеотидные повторы (21.21\%). Праймеры успешно разработаны для 45 (68.18\%) SSR маркеров.

KEYWORDS: Bryophytes, Marchantia, Chloroplast, Microsatellites, Simple Sequence Repeats

\section{INTRODUCTION}

Bryophytes are broadly classified into liverworts, mosses and hornworts, and are considered as the earliest land plants. Chloroplast and mitochondrial genome sequences based phylogenetic analysis showed liverworts as the earliest diverging lineage and hornworts as sister group to vascular plants (Shanker, 2013a,b,c).

Among land plants the first complete chloroplast genome sequence determined was that of Marchantia polymorpha (Ohyama et al., 1986; Ohyama et al., 1988). Since then several studies to elucidate the structure and organization of chloroplast genomes have been conducted. However, a small number of organelle genome sequences of bryophytes are available (Shanker, 2012a,b).

Simple sequence repeats (SSRs) or microsatellites are found in DNA sequences and consist of short repeat motifs of 1-6 bp (Shanker et al., 2007b). These repeats are present in both coding and non-coding regions of DNA sequences, and have been widely used as molecular markers in plant genomes (Gupta et al., 2003; Jakobsson et al., 2007; Blair \& Hurtado, 2013). Studies were conducted to mine SSRs in chloroplast genomes of bryophytes (Shanker, 2013d,e). However, mining of other available organelle genome sequences of bryophytes will help to know the occurrence of SSRs in them.

Biotechnological methods of SSR identification are costly and consume time. Contrary to this bioinformatic approaches offer rapid and economical SSR extraction using sequences deposited in public databases (Shanker et al., 2007a). Therefore the present analysis was conducted to identify cpSSRs in M. polymorpha. Moreover, the distribution of these repeats in coding and non-coding regions of chloroplast genome was analyzed.

1 - Department of Bioscience and Biotechnology, Banasthali University, Banasthali-304022, Rajasthan, India; e-mail: ashomics@gmail.com 


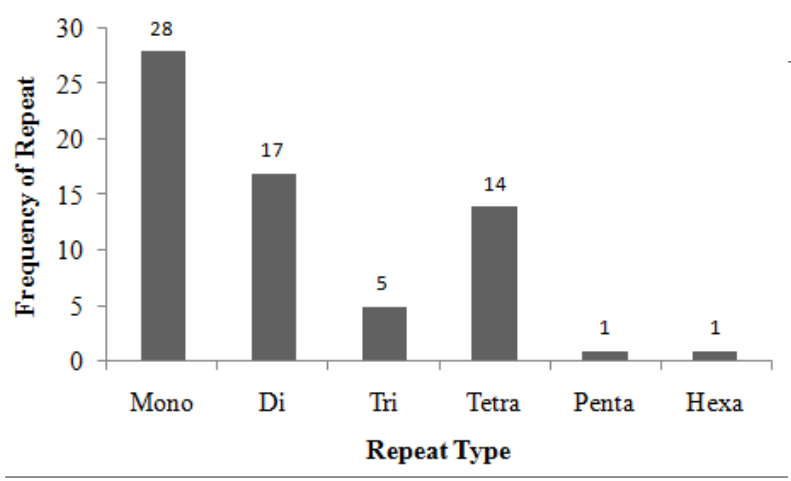

MATERIALS AND METHODS

Chloroplast genome sequence of $M$. polymorpha. The complete chloroplast genome sequence of $M$. polymorpha (NC_001319, 121024 bp; Ohyama et al., 1986) was downloaded from NCBI (www.ncbi.nlm.nih.gov) in FASTA and GenBank format.

Identification of chloroplastic simple sequence repeats. A Perl script named MISA (available at http:// pgrc.ipkgatersleben.de/misa/misa) was used to identify SSRs in chloroplast genome sequence of $M$. polymorpha. MISA takes FASTA formatted DNA sequence file as an input and generates information of perfect and compound SSRs, if found. A perfect SSR consist of same repeating motif without interruptions, e.g., (GTG). In compound SSR, two or more SSRs are found adjacent to one another, e.g., $(\mathrm{GTG})_{8}(\mathrm{AT})_{16}$ (Bachmann \& Bare, 2004). The length of SSRs in this study was defined as $\geq 12$ for mono, di, tri and tetranucleotide, $\geq 15$ for pentanucleotide and $\geq 18$ for hexanucleotide repeats. Maximum difference between two compound SSRs was taken as 0 . Since the information of coding and non-coding regions of chloroplast genome has been available in GenBank file, therefore, the mined cpSSRs were classified as coding, non-coding and coding-non-coding (occurrence of few bases of SSR in coding as well as in non-coding regions or vice-versa) SSRs based on the presence of these repeats in respective regions.

Primer designing for identified SSRs. PCR primers were designed for identified SSRs considering 200 base pair of SSR flanking regions using Primer 3 (http://bioinfo.ut.ee/ primer3-0.4.0/) with default parameters of GC content, melting temperature, primer and PCR product size.

\section{RESULTS AND DISCUSSION}

In this study SSRs were identified in chloroplast genome sequence of $M$. polymorpha considering a minimum length of $12 \mathrm{bp}$. A total of 66 perfect SSRs were detected with length variation from 12 to $64 \mathrm{bp}$. Compound SSRs were found to be totally absent in chloroplast genome sequence of $M$. polymorpha. The frequency of identified SSRs (mono-hexa) is presented in Fig. 1. Detail information of mined SSRs motif, their length, start-end position and the region in which they lie is presented in Table 1. It is evident from this table that out of total cpSSRs detected, 14 (21.21\%) found in coding, 48 $(72.73 \%)$ in non-coding and only $4(6.06 \%)$ in coding-
Fig. 1. Frequency distribution of mono-hexa repeats.

non-coding regions. The occurrence of these repeats in various regions attributed to the evolutionary processes that fine tune distribution of SSR repeat types in genome (Lin \& Kussell, 2012).

Mononucleotides were found to be most frequent repeat $(28,42.42 \%)$ followed by dinucleotide (17, $25.76 \%)$, tetranucleotide $(14,21.21 \%)$ and trinucleotide $(5,7.58 \%)$ repeats. Penta and hexanucleotide repeats were found with equal frequencies $(1,1.52 \%)$. An attempt was made to design PCR primers for all mined SSRs, however, these were successfully designed for 45 $(68.18 \%)$ cpSSRs of $M$. polymorpha. A list of designed PCR primers, their length, product size etc. is presented in Table 2.

The mined SSRs represent a density of $1 \mathrm{SSR} / 1.83$ $\mathrm{kb}$ in $121.024 \mathrm{~kb}$ sequence mined. The density of cpSSRs in $M$. polymorpha found to be higher than the density of cpSSRs in Anthoceros formosae (1 SSR/2.4 kb; Shanker, 2013d), rice (1 SSR/6.5 kb; Rajendrakumar et al., 2007), EST-SSRs in barley, maize, wheat, rye, sorghum and rice (1 SSR/6.0 kb; Varshney et al., 2002), cotton and poplar (1 SSR/20 kb and $1 \mathrm{SSR} / 14 \mathrm{~kb}$ respectively; Cardle et al.,, 2000), Unigenes sequences of Citrus (1 SSR/12.9 $\mathrm{kb}$; Shanker et al., 2007a). However, the density of cpSSRs in $M$. polymorpha found to be lower than the cpSSRs density in family Solanaceae (1 SSR/1.26kb; Tambarussi et al., 2009). The selection of SSR detection tools, parameters taken (e.g. minimum length of SSRs) and amount of data analyzed might be the cause of variations in SSR density.

The higher occurrence of mononucleotides in this study shows consistency with earlier studies of cpSSRs in Anthoceros formosae (Shanker, 2013d), rice (Rajendrakumar et al., 2007), Solanaceae species (Tambarussi et al., 2009), Saccharum spp. (Melotto-Passarin et al., 2011) and Olea species (Filiz \& Koc, 2012). Moreover the abundance of cpSSRs in non-coding regions is also in agreement with previous studies (Hancock, 1995; Shanker, 2013d).

As a concluding remark, in silico mining of complete chloroplast genome sequence of $M$. polymorpha saves time, cost and provides sufficient number of SSRs for this liverwort. The identified SSRs can be used to develop SSR markers and for various other purposes.

\section{LITERATIRE CITED}

BACHMANN, L. \& P.T.J. BARE 2004. Allelic variation, fragment length analyses and population genetic model: A case study on Drosophila microsatellites. - J. Zool. Syst. Evol. Research 42: 215-222.

BLAIR, M.W. \& N. HURTADO 2013. EST-SSR markers from five sequenced cDNA libraries of common bean (Phaseolus vulgaris L.) comparing three bioinformatic algorithms. - Mol. Ecol. Resour. 13: 688-695.

CARDLE, L., L. RAMSAY, D. MILBOURNE, M. MACAULAY, D. MARSHALL \& R. WAUGH 2000. Computational and experimental characterization of physically clustered simple sequence repeats in plants. - Genetics 156: 847-854. 


\begin{tabular}{|c|c|c|c|c|}
\hline MOTIF L & ENGTH & START & END & REGION \\
\hline (ATAAA)3 & 15 & 450 & 464 & Non coding \\
\hline (AT)7 & 14 & 2290 & 2303 & Non coding \\
\hline (TAA)4 & 12 & 2510 & 2521 & Non coding \\
\hline (TA) 32 & 64 & 3589 & 3652 & Non coding \\
\hline$(\mathrm{AAC}) 7$ & 21 & 4559 & 4579 & Non coding \\
\hline (AT)6 & 12 & 5318 & 5329 & Non coding \\
\hline (TA) 17 & 34 & 5379 & 5412 & Non coding \\
\hline (T) 12 & 12 & 5491 & 5502 & Non coding \\
\hline (A) 14 & 14 & 7640 & 7653 & Coding \\
\hline (T) 14 & 14 & 9981 & 9994 & Non coding \\
\hline (A) 15 & 15 & 13493 & 13507 & Coding \\
\hline (A) 12 & 12 & 14664 & 14675 & Coding \\
\hline (A) 13 & 13 & 15824 & 15836 & Coding \\
\hline (T) 12 & 12 & 22002 & 22013 & Non coding \\
\hline (AT) 24 & 48 & 22693 & 22740 & Non coding \\
\hline (AAT)6 & 18 & 23325 & 23342 & Non coding \\
\hline (A) 13 & 13 & 23923 & 23935 & Non coding \\
\hline (T) 13 & 13 & 26530 & 26542 & Non coding \\
\hline$(\mathrm{AAAC}) 3$ & 12 & 26632 & 26643 & Non coding \\
\hline$(\mathrm{AT}) 7$ & 14 & 26664 & 26677 & Non coding \\
\hline (T) 12 & 12 & 26745 & 26756 & Non coding \\
\hline (A) 15 & 15 & 30115 & 30129 & Coding \\
\hline (A) 13 & 13 & 33266 & 33278 & Coding \\
\hline (ATTA) 3 & 12 & 36685 & 36696 & Non coding \\
\hline (TA) 8 & 16 & 38168 & 38183 & Non coding \\
\hline (A) 13 & 13 & 38339 & 38351 & Non coding \\
\hline (TTAA)6 & 24 & 41278 & 41301 & Coding-Non-Coding \\
\hline (A) 12 & 12 & 41323 & 41334 & Non coding \\
\hline (TA) 13 & 26 & 41988 & 42013 & Non coding \\
\hline (A) 16 & 16 & 50459 & 50474 & Non coding \\
\hline (TAA)4 & 12 & 50760 & 50771 & Non coding \\
\hline$(\mathrm{AT}) 6$ & 12 & 53692 & 53703 & Non coding \\
\hline (ATTA)3 & 12 & 53937 & 53948 & Non coding \\
\hline$(\mathrm{TA}) 8$ & 16 & 56040 & 56055 & Non coding \\
\hline (AT) 16 & 32 & 56076 & 56107 & Non coding \\
\hline (A) 12 & 12 & 58041 & 58052 & Non coding \\
\hline (TTA)4 & 12 & 59082 & 59093 & Non coding \\
\hline (A) 13 & 13 & 60479 & 60491 & Coding \\
\hline (TTTG)3 & 12 & 63125 & 63136 & Coding \\
\hline (T)12 & 12 & 63943 & 63954 & Non coding \\
\hline (TA) 6 & 12 & 64048 & 64059 & Non coding \\
\hline (A) 16 & 16 & 64355 & 64370 & Coding-Non-Coding \\
\hline (TTATAT) 3 & 18 & 64657 & 64674 & Non coding \\
\hline (AT)6 & 12 & 64681 & 64692 & Non coding \\
\hline (T) 13 & 13 & 64817 & 64829 & Non coding \\
\hline (TA) 6 & 12 & 64853 & 64864 & Non coding \\
\hline$(\mathrm{TA}) 7$ & 14 & 67473 & 67486 & Non coding \\
\hline (TATT)4 & 16 & 68702 & 68717 & Non coding \\
\hline (TAAA) 3 & 12 & 70571 & 70582 & Non coding \\
\hline (T) 13 & 13 & 72980 & 72992 & Non coding \\
\hline (T) 12 & 12 & 75959 & 75970 & Coding \\
\hline (TTTA) 3 & 12 & 76223 & 76234 & Non coding \\
\hline (T) 15 & 15 & 76621 & 76635 & Coding-Non-Coding \\
\hline (T) 13 & 13 & 77537 & 77549 & Non coding \\
\hline (TTTA)3 & 12 & 79756 & 79767 & Non coding \\
\hline (T) 13 & 13 & 79811 & 79823 & Non coding \\
\hline (T) 17 & 17 & 80642 & 80658 & Coding \\
\hline$(\mathrm{AGGT}) 3$ & 12 & 87572 & 87583 & Coding \\
\hline (TTTA) 3 & 12 & 95400 & 95411 & Non coding \\
\hline (AATA)3 & 12 & 96864 & 96875 & Coding \\
\hline (AT)6 & 12 & 98203 & 98214 & Non coding \\
\hline$(\mathrm{AT}) 7$ & 14 & 98579 & 98592 & Non coding \\
\hline (A) 16 & 16 & 99738 & 99753 & Non coding \\
\hline$(\mathrm{AATT}) 3$ & 12 & 99773 & 99784 & Coding-Non-Coding \\
\hline (T) 14 & 14 & 106439 & 106452 & Coding \\
\hline (CTAC) 3 & 12 & 114535 & 114546 & Coding \\
\hline
\end{tabular}

Table 1: Information of mined SSRs in chloroplast genome sequence of M. polymorpha.

FILIZ, E \& I. KOC 2012. In silico chloroplast SSRs mining of Olea species. - Biodiversitas 13: 114-117.

GUPTA, P.K., S. RUSTGI, S. SHARMA, R. SINGH, N. KUMAR \& H.S. BALYAN 2003. Transferable EST-SSR markers for the study of polymorphism and genetic diversity in bread wheat. -Mol. Genet. Genomics 270: 315-323.

HANCOCK, J.M. 1995. The contribution of slippage-like processes to genome evolution. - J. Mol. Evol. 41: 1038-1047.

JAKOBSSON, M., T. SALL, C. LIND-HALLDEN \& C. HALLDEN 2007. Evolution of chloroplast mononucleotide microsatellites in Arabidopsis thaliana. - Theor. Appl. Genet. 114: 223-235.

LIN, W.H. \& E. KUSSELL 2012. Evolutionary pressures on simple sequence repeats in prokaryotic coding regions. - Nucleic Acids Res. 40: 2399-2413.

MELOTTO-PASSARIN, D.M., E.V. TAMBARUSSI, K. DRESSANO, V.F. DE MARTIN \& H. CARRER 2011. Characterization of chloroplast DNA microsatellites from Saccharum spp. and related species. Genet. Mol. Res. 10: 2024-2033.

OHYAMA, K., H. FUKUZAWA, T. KOHCHI, H. SHIRAI, T. SANO, S. SANO, K. UMESONO, Y. SHIKI, M. TAKEUCHI, Z. CHANG, S. AOTA, H. INOKUCHI \& H. OZEKI 1986. Chloroplast gene organization deduced from complete sequence of liverwort Marchantia polymorpha chloroplast DNA. - Nature 322: 572-574.

OHYAMA, K., H. FUKUZAWA, T. KOHCHI, T. SANO, S. SANO, H. SHIRAI, K. UMESONO, Y. SHIKI, M. TAKEUCHI, Z. CHANG, H. INOKUCHI \& H. OZEKI 1988. Structure and organization of Marchantia polymorpha chloroplast genome. I. Cloning and gene identification. - J. Mol. Biol. 203: 281-298.

RAJENDRAKUMAR, P., A.K. BISWAL, S.M. BALACHANDRAN, K. SRINIVASARAO \& R.M. SUNDARAM 2007. Simple sequence repeats in organellar genomes of rice: frequency and distribution in genic and intergenic regions. - Bioinformatics 23: 1-4.

SHANKER, A. 2012a. Chloroplast genomes of bryophytes: a review. Archive for Bryology 143: 1-5.

SHANKER, A. 2012b. Sequenced mitochondrial genomes of bryophytes. - Archive for Bryology 146: 1-6.

SHANKER, A. 2013a. Paraphyly of bryophytes inferred using chloroplast sequences. - Archive for Bryology 163: 1-5.

SHANKER, A. 2013b. Inference of bryophytes paraphyly using mitochondrial genomes. - Archive for Bryology 165: 1-5.

SHANKER, A. 2013c. Combined data from chloroplast and mitochondrial genome sequences showed paraphyly of bryophytes. - Archive for Bryology 171: 1-9.

SHANKER, A. 2013d. Identification of microsatellites in chloroplast genome of Anthoceros formosae. - Archive for Bryology 191: 1-6.

SHANKER, A. 2013e. Mining of simple sequence repeats in chloroplast genome of a parasitic liverwort: Aneura mirabilis. - Archive for Bryology 196: 1-4.

SHANKER, A., A. BHARGAV, R. BAJPAI, S. SINGH, S. SRIVASTAVA \& V. SHARMA 2007a. Bioinformatically mined simple sequence repeats in UniGene of Citrus sinensis. - Sci. Hort. 113: 353-361.

SHANKER, A., A. SINGH \& V. SHARMA 2007b. In silico mining in expressed sequences of Neurospora crassa for identification and abundance of microsatellites. - Microbiol. Res. 162: 250-256.

TAMBARUSSI, E.V., D.M. MELOTTO-PASSARIN, S.G. GONZALEZ, J.B. BRIGATI, F.A. DE JESUS, A.L. BARBOSA, K. DRESSANO \& H. CARRER 2009. In silico analysis of simple sequence repeats from chloroplast genomes of Solanaceae species. - Crop Breed. Appl. Biotech. 9: 344-352.

VARSHNEY, R.K., T. THIEL, N. STEIN, P. LANGRIDGE \& A. GRANER 2002. In silico analysis on frequency and distribution of microsatellites in ESTs of some cereal species. - Cell \& Mol. Biol. Lett. 7: 537-546. 
Table 2: List of primers designed for identified SSRs along with primer length, melting temperature and product size.

\begin{tabular}{|c|c|c|c|c|c|c|c|c|}
\hline No. & MOTIF & START & END & LEFT / RIGHT PRIMER & $\begin{array}{l}\text { PRIMER } \\
\text { LENGTH }\end{array}$ & $\mathrm{Tm}$ & $\mathrm{GC} \%$ & $\begin{array}{l}\text { PRODUC } \\
\text { SIZE (bp }\end{array}$ \\
\hline 1 & $(\mathrm{AT}) 7$ & 2290 & 2303 & $\begin{array}{l}\text { ATGAAGGAGTGCGATTCGTT } \\
\text { AAAAATTAATTGGTGAAATTTTGCGTT }\end{array}$ & $\begin{array}{l}20 \\
27\end{array}$ & $\begin{array}{l}57.613 \\
57.205\end{array}$ & $\begin{array}{l}45 \\
22.222\end{array}$ & 152 \\
\hline 2 & (TAA)4 & 2510 & 2521 & $\begin{array}{c}\text { AACGCAAAATTTCACCAATTAATTTTT } \\
\text { TCGAAGAATCCTTCTATTTCAATGGT }\end{array}$ & $\begin{array}{l}27 \\
26\end{array}$ & $\begin{array}{l}57.205 \\
58.661\end{array}$ & $\begin{array}{l}22.222 \\
34.615\end{array}$ & 261 \\
\hline 3 & (TA) 17 & 5379 & 5412 & $\begin{array}{l}\text { GCCCAGGCTATATTAATAATATCCATT } \\
\text { AGACAACCCATCATAAGATTAAAACA }\end{array}$ & $\begin{array}{l}27 \\
26\end{array}$ & $\begin{array}{l}57.186 \\
57.122\end{array}$ & $\begin{array}{l}33.333 \\
30.769\end{array}$ & 300 \\
\hline 4 & (A) 14 & 7640 & 7653 & $\begin{array}{l}\text { ACTGTCTCATCGCATGGAGG } \\
\text { AGCAGCTCCGTCTGCTAAAA }\end{array}$ & $\begin{array}{l}20 \\
20\end{array}$ & $\begin{array}{l}59.537 \\
59.678\end{array}$ & $\begin{array}{l}55 \\
50\end{array}$ & 196 \\
\hline 5 & $(\mathrm{~T}) 14$ & 9981 & 9994 & $\begin{array}{l}\text { TCGAAATAGAGCAAAAACCTAAAGGA } \\
\text { CGTCATCCGGCTCAAATAGT }\end{array}$ & $\begin{array}{l}26 \\
20\end{array}$ & $\begin{array}{l}59.062 \\
57.775\end{array}$ & $\begin{array}{l}34.615 \\
50\end{array}$ & 250 \\
\hline 6 & (AT) 24 & 22693 & 22740 & $\begin{array}{l}\text { TGACCTCAATTTCAGATAGTCAAATT } \\
\text { CACTCGGCCATCTCTCCAAA }\end{array}$ & $\begin{array}{l}26 \\
20\end{array}$ & $\begin{array}{l}57.131 \\
59.75\end{array}$ & $\begin{array}{l}30.769 \\
55\end{array}$ & 393 \\
\hline 7 & (AAT)6 & 23325 & 23342 & $\begin{array}{r}\text { TGCAATTGATTACTACTTTGCCA } \\
\text { GCCTTTGTATGGCAAGCTTCA }\end{array}$ & $\begin{array}{l}23 \\
21\end{array}$ & $\begin{array}{l}57.026 \\
59.454\end{array}$ & $\begin{array}{l}34.783 \\
47.619\end{array}$ & 220 \\
\hline 8 & (A) 13 & 23923 & 23935 & $\begin{array}{l}\text { CGAATCCTTCCGTCCCAGAC } \\
\text { GCAGGACCAGCATACATCCA }\end{array}$ & $\begin{array}{l}20 \\
20\end{array}$ & $\begin{array}{l}60.179 \\
59.819\end{array}$ & $\begin{array}{l}60 \\
55\end{array}$ & 231 \\
\hline 9 & $(\mathrm{~T}) 13$ & 26530 & 26542 & $\begin{array}{l}\text { TGTGTCTAGAAATAACCAGCATGC } \\
\text { TGTTTGTTTGTTTTTCAAAAGTAGGCA }\end{array}$ & $\begin{array}{l}24 \\
27\end{array}$ & $\begin{array}{l}59.364 \\
59.773\end{array}$ & $\begin{array}{l}41.667 \\
29.63\end{array}$ & 192 \\
\hline 10 & $(\mathrm{AAAC}) 3$ & 26632 & 26643 & $\begin{array}{l}\text { AGAAATAACCAGCATGCTGATTAAT } \\
\text { TGCAATTCCATAAAAATTTTCCTGAA }\end{array}$ & $\begin{array}{l}25 \\
26\end{array}$ & $\begin{array}{l}57.407 \\
57.085\end{array}$ & $\begin{array}{l}32 \\
26.923\end{array}$ & 250 \\
\hline 11 & $(\mathrm{AT}) 7$ & 26664 & 26677 & $\begin{array}{l}\text { AAAGTTTTGCCTACTTTTGAAAAACA } \\
\text { TGCAATTCCATAAAAATTTTCCTGAA }\end{array}$ & $\begin{array}{l}26 \\
26\end{array}$ & $\begin{array}{l}57.615 \\
57.085\end{array}$ & $\begin{array}{l}26.923 \\
26.923\end{array}$ & 100 \\
\hline 12 & (ATTA) 3 & 36685 & 36696 & $\begin{array}{l}\text { CCAGCGGATTTACAGTCCGT } \\
\text { TCTCTTTCAAGGAGGCGACG }\end{array}$ & $\begin{array}{l}20 \\
20\end{array}$ & $\begin{array}{l}60.108 \\
59.756\end{array}$ & $\begin{array}{l}55 \\
55\end{array}$ & 163 \\
\hline 13 & $(\mathrm{TA}) 8$ & 38168 & 38183 & $\begin{array}{l}\text { TCCAATAGGTTATCAATCTTTCCGA } \\
\text { CACTGAGTTAAAAGGGCAATAAGA }\end{array}$ & $\begin{array}{l}25 \\
24\end{array}$ & $\begin{array}{l}57.868 \\
57.182\end{array}$ & $\begin{array}{l}36 \\
37.5\end{array}$ & 387 \\
\hline 14 & (TTAA) 6 & 41278 & 41301 & $\begin{array}{l}\text { CTGCTGCAGCTGGTTTTGAA } \\
\text { TCGAGGGTTCAAATCCCTCTC }\end{array}$ & $\begin{array}{l}20 \\
21\end{array}$ & $\begin{array}{l}59.614 \\
59.168\end{array}$ & $\begin{array}{l}50 \\
52.381\end{array}$ & 223 \\
\hline 15 & (A) 12 & 41323 & 41334 & $\begin{array}{l}\text { CTGCTGCAGCTGGTTTTGAA } \\
\text { TCGAGGGTTCAAATCCCTCTC }\end{array}$ & $\begin{array}{l}20 \\
21\end{array}$ & $\begin{array}{l}59.614 \\
59.168\end{array}$ & $\begin{array}{l}50 \\
52.381\end{array}$ & 223 \\
\hline 16 & (A) 16 & 50459 & 50474 & $\begin{array}{l}\text { GCATTACAAGTGCGACGCTC } \\
\text { CCAATTTCGCCATACCCCCT }\end{array}$ & $\begin{array}{l}20 \\
20\end{array}$ & $\begin{array}{l}60.247 \\
60.106\end{array}$ & $\begin{array}{l}55 \\
55\end{array}$ & 249 \\
\hline 17 & (TAA) 4 & 50760 & 50771 & $\begin{array}{c}\text { ACAAAAGAGGTGCAGAGACTCA } \\
\text { CTGGGGGTAGAGGGACTTGA }\end{array}$ & $\begin{array}{l}22 \\
20\end{array}$ & $\begin{array}{l}59.564 \\
59.957\end{array}$ & $\begin{array}{l}45.455 \\
60\end{array}$ & 248 \\
\hline 18 & (AT)6 & 53692 & 53703 & $\begin{array}{l}\text { TACCGCTGAGCTATAGCCCT } \\
\text { CTCTCGCCGTATGAAAGCGA }\end{array}$ & $\begin{array}{l}20 \\
20\end{array}$ & $\begin{array}{l}59.887 \\
60.248\end{array}$ & $\begin{array}{l}55 \\
55\end{array}$ & 216 \\
\hline 19 & (ATTA)3 & 53937 & 53948 & $\begin{array}{l}\text { TCGCTTTCATACGGCGAGAG } \\
\text { AGAGCTAAAGCAAGATTAGAAGCA }\end{array}$ & $\begin{array}{l}20 \\
24\end{array}$ & $\begin{array}{l}60.248 \\
58.261\end{array}$ & $\begin{array}{l}55 \\
37.5\end{array}$ & 176 \\
\hline 20 & (A) 12 & 58041 & 58052 & $\begin{array}{l}\text { TGGGTTTGTAGCTCAGTGGA } \\
\text { AGCGCCAATTAATCCACCAA }\end{array}$ & $\begin{array}{l}20 \\
20\end{array}$ & $\begin{array}{l}58.57 \\
58.154\end{array}$ & $\begin{array}{l}50 \\
45\end{array}$ & 247 \\
\hline 21 & (TTA)4 & 59082 & 59093 & $\begin{array}{l}\text { TGAATTATATAACGCTGCTCCTTGT } \\
\text { GCAGGAAAAATTAATCCAACTAAAGGA }\end{array}$ & $\begin{array}{l}25 \\
27\end{array}$ & $\begin{array}{l}58.712 \\
58.689\end{array}$ & $\begin{array}{l}36 \\
33.333\end{array}$ & 292 \\
\hline 22 & (A) 13 & 60479 & 60491 & $\begin{array}{l}\text { TCAAAACGTTCTTGGCAGTCT } \\
\text { AGCTCTAATCCAAGCTAATTTTCTGT }\end{array}$ & $\begin{array}{l}21 \\
26\end{array}$ & $\begin{array}{l}58.361 \\
58.885\end{array}$ & $\begin{array}{l}42.857 \\
34.615\end{array}$ & 235 \\
\hline 23 & (TTTG)3 & 63125 & 63136 & $\begin{array}{l}\text { ACCCCAATAGAGGCTAGTACGA } \\
\text { ACCGTAAGATGGTTAGCCGT }\end{array}$ & $\begin{array}{l}22 \\
20\end{array}$ & $\begin{array}{l}59.824 \\
58.811\end{array}$ & $\begin{array}{l}50 \\
50\end{array}$ & 175 \\
\hline 24 & $(\mathrm{TA}) 6$ & 64048 & 64059 & $\begin{array}{r}\text { TTACAGATTGGTCTATCCCGAAA } \\
\text { ACGCTAATGTTAAAGCACCGA }\end{array}$ & $\begin{array}{l}23 \\
21\end{array}$ & $\begin{array}{l}57.057 \\
58.309\end{array}$ & $\begin{array}{l}39.13 \\
42.857\end{array}$ & 345 \\
\hline 25 & (A) 16 & 64355 & 64370 & $\begin{array}{l}\text { TCGGTGCTTTAACATTAGCGT } \\
\text { TCCAGACAACAAAGCTTCAACC }\end{array}$ & $\begin{array}{l}21 \\
22\end{array}$ & $\begin{array}{l}58.309 \\
59.312\end{array}$ & $\begin{array}{l}42.857 \\
45.455\end{array}$ & 209 \\
\hline 26 & (TTATAT)3 & 64657 & 64674 & $\begin{array}{l}\text { TGGAGACCTACGTTCTACCGA } \\
\text { TGGTAGCGCGTTTGTTTTGG }\end{array}$ & $\begin{array}{l}21 \\
20\end{array}$ & $\begin{array}{l}59.721 \\
59.97\end{array}$ & $\begin{array}{l}52.381 \\
50\end{array}$ & 183 \\
\hline 27 & (AT)6 & 64681 & 64692 & $\begin{array}{l}\text { TGGAGACCTACGTTCTACCGA } \\
\text { TGGTAGCGCGTTTGTTTTGG }\end{array}$ & $\begin{array}{l}21 \\
20\end{array}$ & $\begin{array}{l}59.721 \\
59.97\end{array}$ & $\begin{array}{l}52.381 \\
50\end{array}$ & 183 \\
\hline 28 & $(\mathrm{TA}) 6$ & 64853 & 64864 & $\begin{array}{l}\text { CCAAACTGCGCTACATCCCT } \\
\text { ACAGGTGCAGTAGAAAGATATGT }\end{array}$ & $\begin{array}{l}20 \\
23\end{array}$ & $\begin{array}{l}60.393 \\
57.316\end{array}$ & $\begin{array}{l}55 \\
39.13\end{array}$ & 297 \\
\hline 29 & (TATT) 4 & 68702 & 68717 & $\begin{array}{l}\text { ACTTTCGGAACACCAATAGGCA } \\
\text { ACAATGGAGAGATTGGTCCCA }\end{array}$ & $\begin{array}{l}22 \\
21\end{array}$ & $\begin{array}{l}60.225 \\
58.722\end{array}$ & $\begin{array}{l}45.455 \\
47.619\end{array}$ & 233 \\
\hline
\end{tabular}


Table 2 (cont.): List of primers designed for identified SSRs along with primer length, melting temperature and product size.

\begin{tabular}{|c|c|c|c|c|c|c|c|c|}
\hline No. & MOTIF & START & END & LEFT / RIGHT PRIMER & $\begin{array}{l}\text { PRIMER } \\
\text { LENGTH }\end{array}$ & $\mathrm{Tm}$ & $\mathrm{GC} \%$ & $\begin{array}{l}\text { PRODUCT } \\
\text { SIZE (bp) }\end{array}$ \\
\hline \multirow[t]{2}{*}{30} & (TAAA)3 & 70571 & 70582 & GCTCAAGTGGAATTTGGAGCG & 21 & 60.136 & 52.381 & 232 \\
\hline & & & & TGATTCCTAAAGTACCTACCAACA & 24 & 57.061 & 37.5 & \\
\hline \multirow[t]{2}{*}{31} & $(\mathrm{~T}) 13$ & 72980 & 72992 & TGAATAGTAAAGACTTCGTTAAATCCA & 27 & 57.068 & 29.63 & 280 \\
\hline & & & & TCATCCGGCTCCAACATTAATGA & 23 & 60.118 & 43.478 & \\
\hline \multirow[t]{2}{*}{32} & $(\mathrm{~T}) 12$ & 75959 & 75970 & CCCATTCCACCTAAAACTTTTGGA & 24 & 59.409 & 41.667 & 241 \\
\hline & & & & AACAGTTCAAGTACCTGCTACT & 22 & 57.042 & 40.909 & \\
\hline \multirow[t]{2}{*}{33} & $(\mathrm{TTTA}) 3$ & 76223 & 76234 & TCGCAATTGTATCATTCCCCA & 21 & 57.711 & 42.857 & 192 \\
\hline & & & & AGGAACTCGAGTTTTTGGTCCA & 22 & 59.828 & 45.455 & \\
\hline \multirow[t]{2}{*}{34} & $(\mathrm{~T}) 15$ & 76621 & 76635 & TGGCACTGCTTCTTTAACAACA & 22 & 58.98 & 40.909 & 195 \\
\hline & & & & GGCCCTCCCTAATCCATCCA & 20 & 60.772 & 60 & \\
\hline \multirow[t]{2}{*}{35} & $(\mathrm{~T}) 13$ & 77537 & 77549 & TGCGTTTTAATACTATAACAAAAACCC & 27 & 57.377 & 29.63 & 199 \\
\hline & & & & GCTATGCTTAGTGTGTGACTCG & 22 & 59.145 & 50 & \\
\hline \multirow[t]{2}{*}{36} & $(\mathrm{~T}) 13$ & 79811 & 79823 & TCATTTAGCATCAGAAAGGATGACT & 25 & 58.343 & 36 & 277 \\
\hline & & & & AGGAAAAATAGGCAAGTGAAAAAGGT & 26 & 59.9 & 34.615 & \\
\hline \multirow[t]{2}{*}{37} & $(\mathrm{~T}) 17$ & 80642 & 80658 & ACAGTATATCCTGTTGTCGTACCT & 24 & 58.801 & 41.667 & 212 \\
\hline & & & & TTATGAATCAAGTTAAGTACCCAGTAC & 27 & 57.12 & 33.333 & \\
\hline \multirow[t]{2}{*}{38} & $(\mathrm{AGGT}) 3$ & 87572 & 87583 & CTTCCAGCCAATGTCCGAGT & 20 & 60.036 & 55 & 153 \\
\hline & & & & TTATCTCGCGCCCCTAGGTA & 20 & 59.889 & 55 & \\
\hline \multirow[t]{2}{*}{39} & (TTTA) 3 & 95400 & 95411 & AGCAGTGCTAAGGCTTCTCG & 20 & 60.108 & 55 & 194 \\
\hline & & & & ACGCTCTAAGGTTATAAATGGCA & 23 & 57.969 & 39.13 & \\
\hline \multirow[t]{2}{*}{40} & (AATA)3 & 96864 & 96875 & TTTCTCGTGGTCCAGCATCC & 20 & 60.036 & 55 & 227 \\
\hline & & & & GCTTTGCCAGGTATGAGTGG & 20 & 58.902 & 55 & \\
\hline \multirow[t]{2}{*}{41} & $(\mathrm{AT}) 6$ & 98203 & 98214 & ACGGGATTACTAAACCTGCAGA & 22 & 59.164 & 45.455 & 226 \\
\hline & & & & TGAGACTACTCGTAGTATGGGTCT & 24 & 59.592 & 45.833 & \\
\hline \multirow[t]{2}{*}{42} & (A) 16 & 99738 & 99753 & TCTGGTAATTTCATAAAGCTCACAA & 25 & 57.006 & 32 & 165 \\
\hline & & & & CCTAAAGGCAAAATAGAAGGGCA & 23 & 58.977 & 43.478 & \\
\hline \multirow[t]{2}{*}{43} & $(\mathrm{AATT}) 3$ & 99773 & 99784 & TCTGGTAATTTCATAAAGCTCACAA & 25 & 57.006 & 32 & 165 \\
\hline & & & & CCTAAAGGCAAAATAGAAGGGCA & 23 & 58.977 & 43.478 & \\
\hline \multirow[t]{2}{*}{44} & $(\mathrm{~T}) 14$ & 106439 & 106452 & TGGAAAATCCCATCTGTTTGCT & 22 & 58.491 & 40.909 & 248 \\
\hline & & & & AGGATCTATGCGTGCTCGAC & 20 & 59.688 & 55 & \\
\hline \multirow[t]{2}{*}{45} & $(\mathrm{CTAC}) 3$ & 114535 & 114546 & TTATCTCGCGCCCCTAGGTA & 20 & 59.889 & 55 & 153 \\
\hline & & & & CTTCCAGCCAATGTCCGAGT & 20 & 60.036 & 55 & \\
\hline
\end{tabular}

